\title{
Prophylaxis and Treatment of InVAsive Aspergillosis With Voriconazole, Posaconazole and CASPOFUnGIN - REVIEW OF THE LITERATURE
}

\author{
M. Karthaus
}

Klinikum Neuperlach, München, Germany

\begin{abstract}
Major progress for the management of invasive aspergillosis has come from the introduction of new antifungals since the late 1990s. Although mortality of invasive aspergillosis remains as high as $30-50 \%$. Backbone of management are prophylaxis, early diagnosis and early initiation of antifungals for reduction of invasive aspergillosis related mortality. Randomized trials have been undertaken for the prophylaxis as well as treatment of invasive aspergillosis in the last two decades. Posaconazole is recommended for prophylaxis against aspergillosis in patients treated for acute myelogenous leukemia, myelodysplastic syndrome or patients with graft versus host disease after allogeneic transplantation. Efficacy has been shown for first-line therapy of invasive aspergillosis with voriconazole and liposomal amphotericin B. Gastrointestinal resorption for the azoles posaconazole, voriconazole and itraconazole differ considerably. While oral voriconazole resportion is reduced when taken with food, posaconazole has to be taken with fatty food for optimal intestinal resorption. Beside all advances in the management of invasive aspergillosis important questions remain unresolved. This article reviews the current state of prophylaxis and treatment of invasive aspergillosis and points out clinicians unmet needs.
\end{abstract}

\section{INTRODUCTION}

Fungal infections are an important cause of morbidity for patients with hematological malignancies. The epidemiology of invasive fungal infections has changed within the last decade. While infections due to Candida species continue to be frequent despite a broader use of azoles in the prophylactic setting, infections due to Aspergillus species remain the leading pathogen in the postmortem epidemiology [1]. The genus Aspergillus includes over 185 species. Out of these around 20 have been reported causative of opportunistic infections in man. The manifestation and severity of the aspergillosis disease depends upon the immune status of the patient. Invasive aspergillus infections most commonly affect the lung (see Fig. 1) and sinuses. Other forms of the disease are central nervous aspergillosis, osteomyelitis, endophthalmitis, endocarditis and disseminated form of aspergillosis which are attributed with a morbidity and a high risk of infection related death. Invasive aspergillus infections are rarely observed in healthy hosts [2, 3]. Therefore a thorough knowledge of risk factors, potential causative organisms, and the safety and efficacy of appropriate antifungal agents is required for optimal management. Risk factors for aspergillus infections are outlined in Table $1[4,5]$. Infections due to Aspergillus species are caused in most cases by Aspergillus fumigatus, far ahead of Aspergillus flavus, Aspergillus niger, Aspergillus terreus and other Aspergillus species (Table 2). Species distribution may differ which means that local epidemiology should be

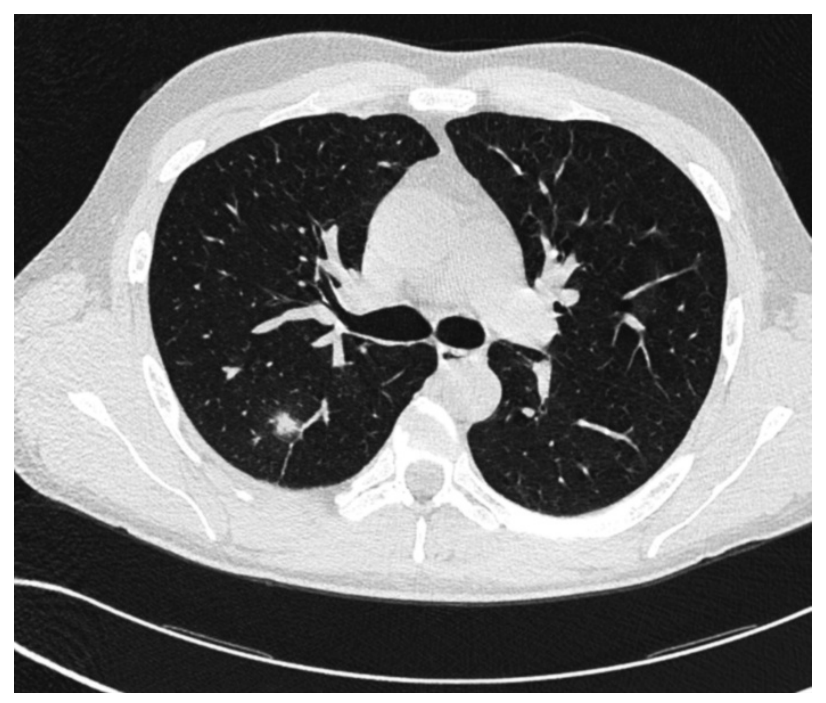

Fig. 1. Pulmonary aspergillosis with a typical halo sign in the right lung.

Table 1. Risk Factors for Invasive Aspergillus Infections.

severe and long lasting neutropenia

bone marrow transplantation

acute and chronic graft-versus-host disease

uncontrolled diabetes

continuous use of steroids

uncontrolled construction work

sustainded immune suppression 
Table 2. Important causative Aspergillus species with Opportunistic Infections in Human.

Aspergillus fumigatus
Aspergillus flavus
Aspergillus niger
Aspergillus nidulans
Aspergillus terreus
Aspergillus versiculor

kept in mind (e.g. A. terreus with a lack of susceptibility against amphotericin B). Definitive diagnosis by culture may take four or more days. Most patients are treated prior to proven diagnosis. A delay or inappropriate treatment have been associated with an inverse outcome of invasive aspergillosis. Early diagnosis of invasive fungal infection remains a challenge and is of utmost importance. The detection of serum galactomannan (GM) antigen allows 5-8 days earlier diagnosis of invasive aspergillosis when compared to clinical signs, imaging or even cultures of Aspergillus species alone [6]. Platelia GM Aspergillus EIA is a commercially available test kit that detects an exoantigen of Aspergillus with a high sensitivity and specificity of $>80 \%$ and $90 \%$ respectively which may trigger an early treatment initiation against invasive aspergillosis. Given the high mortality associated with invasive aspergillosis prophylaxis of invasive fungal infections would be ideal [7]. Antifungal therapy is associated with adverse events and has a substantial economic burden in addition. These aspects must be taken into account. The numbers needed to prevent an invasive aspergillosis differ considerably between institutions. Environmental situations are only one reason, beside patients and their risk factors for invasive aspergillosis. Early preemptive treatment with a safe antifungal agent would be an alternative approach, if early diagnosis and effective treatment could reliably be established.

\section{Antifungal DRUgS}

Polyenes were introduced for antifungal treatment in the late 1950s. Amphotericin B desoxycholate (AmB) is proven to be effective but toxic. AmB has a lot of severe adverse events including nephrotoxicity and infusion related side effects. Although prolongation of infusion time from two to four and up to $24 \mathrm{~h}$ has been shown to be less toxic [8], recent guidelines have dumped amphotericin B desoxycholate for prophylaxis and empirical treatment $[9,10]$. Three lipid based amphotericin B formulations have been introduced since the 1990s. Out of these, liposomal amphotericin B (LAmB) has proved to overcome a substantial number of adverse events. LAmB is significantly less nephrotoxic and associated with fewer infusion-related side effects. Randomized trials with LAmB have shown efficacy comparable with amphotericin B desoxycholate for empirical antifungal therapy in patients with febrile neutropenia refractory to broad-spectrum antibiotics $[11,12]$. New azoles were introduced with susceptibility against Aspergillus species. These second generation azoles have been studied for prophylaxis and treatment since the late 1990s. Voriconazole and posaconazole proved efficacy. Voriconazole is approved for 1st-line treatment of invasive aspergillosis, while posaconazole is approved for prophylaxis of invasive fungal infections and 2nd-line treatment of invasive aspergillosis.

Echinocandins are a new class of antifungals. Echinocandins inhibit the synthesis of $1,3-\beta$-D-glucan, an essential component of the fungal cell wall. They exhibit potent in vitro and in vivo antifungal activity against Candida and Aspergillus species. Caspofungin, Micafungin and Anidulafungin are approved for antifungal treatment in the EU. There was no substantial difference in efficacy with liposomal amphotericin B in comparison with caspofungin in a randomized trial for treatment of febrile neutropenia [13]. Nephrotoxicity and adverse events were observed less frequent in patients treated with caspofungin. With the introduction of these new and safer antifungals with a proven efficacy against aspergillosis prophylaxis has become a major issue and reasonable field for clinical investigation.

\section{Current Options FOr Prophylaxis of INVASIVE ASPERGILLOSIS}

Fluconazole was introduced for prophylaxis of invasive fungal infections in the early 1990s. Two randomized, placebo-controlled trials showed that fluconazole (400 mg/d) administered prophylactically decreased the incidence of candidiasis in blood and marrow transplant (BMT) recipients [14, 15]. In allogeneic bone marrow transplant (BMT) recipients the use of fluconazole $(400 \mathrm{mg} / \mathrm{d})$ administered over a period of 75 days following BMT resulted in a decreased rate of gut graft versus host disease (GvHD) and a persistent protection against disseminated Candida infections and candidiasis-related death in addition to an overall survival benefit [16]. Fluconazole has major drawbacks due to a growing emergence of non-albicans species with reduced fluconazole susceptibility and what is more important a lack of efficacy against moulds as Aspergillus species. Targeted prophylaxis against aspergillosis is therefore a need and can not be covered by fluconazole.

Two randomized controlled trials evaluated the prophylactic use of posaconazole in acute leukemia and in the allogeneic hematopoietic stem cell transplant (HSCT) setting $[17,18]$. A total of 304 patients with acute myelogenous leukemia (AML) or myelodysplastic syndrome (MDS) that had a chemotherapy-associated neutropenia were treated in a randomized, multicenter study comparing posaconazole $(3 \times 200 \mathrm{mg} / \mathrm{d})$ with fluconazole $(1 \times 400 \mathrm{mg})$ or itraconazole $(2 \times 200$ $\mathrm{mg}$ ) as antifungal prophylaxis. Primary endpoint was the incidence of proven or probable invasive fungal infections during treatment. Proven or probable invasive fungal infections were reported in 7 patients $(2 \%)$ in the posaconazole group and 25 patients $(8 \%)$ in the fluconazole or itraconazole group $(\mathrm{P}<0.001)$. Significantly fewer patients in the posaconazole group had invasive aspergillosis (2 [1\%] vs. 20 [7\%], P<0.001). In a second trial, oral posaconazole was compared with fluconazole p.o. for prophylaxis in patients with graft- 
versus-host disease (GvHD). The primary end point was the incidence of proven or probable invasive fungal infections from randomization to day 112 of the fixed treatment period of the study. In the posaconazole group were fewer breakthrough invasive fungal infections $(2.4 \%$ vs. $7.6 \%, \mathrm{P}=0.004)$, particularly invasive aspergillosis $(1.0 \%$ vs. $5.9 \%, \mathrm{P}=0.001)$. Although overall mortality was similar in the two groups, the number of deaths from invasive fungal infections was reported lower under posaconazole $(1 \%$, vs. $4 \%$; $\mathrm{P}=0.046)$. Both posaconazole trials proved to be effective in the reduction of invasive fungal infections in particular against aspergillosis.

Three glucan synthesis inhibitors were studied for prophylaxis and/or for treatment of invasive fungal infections. Out of these three echinocandins, micafungin has been studied for prophylaxis in the transplant setting. In a randomized, double-blind, phase III trial, 882 adult and pediatric patients received $50 \mathrm{mg}$ of $\mathrm{mi}-$ cafungin i.v. $(1 \mathrm{mg} / \mathrm{kg}$ in pts $<50 \mathrm{~kg})$ or $400 \mathrm{mg}$ fluconazole $(8 \mathrm{mg} / \mathrm{kg}$ in pts $<50 \mathrm{~kg})$ administered once daily. The overall efficacy defined as absence of proven, probable, or suspected systemic fungal infection through the end of prophylaxis with micafungin was superior to fluconazole as during the neutropenic period after HSCT $(\mathrm{P}=.03)$ [19].

Liposomal amphotericin $\mathrm{B}$ is a lipid formulation of amphotericin B with hydrogenated soy phosphatidylcholine, distearoylphophatidylglycerol, and cholesterol. The lipid formulation was developed to obtain a lower toxicity. LAmB was studied in a randomized, unblinded trial comparing intravenous LAmB prophylaxis with no systemic antifungal prophylaxis during neutropenia $(50 \mathrm{mg}$ of $\mathrm{LAmB}$ as a 1 -h infusion every other day) [20]. Invasive aspergillosis occurred less frequently in patients receiving LAmB-prophylaxis $(\mathrm{P}=$ $0.0057)$, whereas the reduction of invasive candidiasis did not reach statistical significance $(\mathrm{P}=0.0655)$. Liposomal amphotericin has been compared to placebo by using an inhalative setting (twice a week) in $271 \mathrm{pa}$ tients with 407 neutropenic episodes ( $\geq 10$ days). The primary end point was occurrence of invasive pulmonary aspergillosis (IPA). Six patients with LAmB prophylaxis versus 18 placebo patients of developed IPA $(\mathrm{P}=.005)$ [21].

In recent years several attempts have been undertaken to prove the efficacy of voriconazole for prophylaxis of invasive fungal infections in hematology malignancies. Studies were done in the transplant and non transplant setting [22]. In a comparative study with allogeneic HSCT-recipients the rates of invasive fungal infections at 6 months were $6.6 \%$ in the voriconazole and $10.6 \%$ in the fluconazole arm. There was a trend towards fewer Aspergillus infections in the voriconazole arm without being superior to fluconazole $(p=0.11)$. In addition overall survival at day +180 was not different between both arms (81\% vs 80\%)[23]. Trials so far had not the design to show differences between voriconazole and fluconazole according to antifungal prophylaxis of invasive aspergillosis, because they didn't compare the same endpoint of treatment. The conceivable value of voriconazole for breakthrough fungal infection after posaconazole prophylaxis is not yet studied in prospective controlled trials.
Itraconazole has been available since the early 1990s. It was studied extensively in the 1990s for prophylaxis of invasive fungal infections. It has been available as oral capsules (since 1992), oral solution in cyclodextrin (since 1997) and intravenous formulation (since 1999). Itraconazole is highly lipophilic. Intestinal absorption of itraconazole oral capsules is variable and unpredictable. Oral solution of itraconazole has a better bioavailability compared to capsules. Most single trials did not prove efficacy against invasive aspergillosis infections. Adverse events, intestinal absorption as well as interactions are a major drawback of oral itraconazole when used for prophylaxis. A meta-analysis showed superiority of itraconazole when compared with fluconazole in hematological malignancies. The incidence of invasive Aspergillus infections was only reduced in trials using the itraconazole cyclodextrine solution and not itraconazole capsules. The overall mortality was not reduced [24].

\section{EVIDENCE BASED RECOMMENDATIONS FOR Aspergillus Prophylaxis}

Infectious disease guidelines recommend prophylaxis against aspergillosis on data of randomized controlled trials. Posaconazole $600 \mathrm{mg} / \mathrm{d}$ is strongly recommended in patients with acute myelogenous leukemia/myelodysplastic syndromes or undergoing allogeneic stem cell recipients with graft versus host disease for the prevention of invasive fungal infections and attributable mortality of invasive aspergillosis (Level A I). Fluconazole $400 \mathrm{mg} / \mathrm{d}$ is recommended in allogeneic stem cell recipients until development of graft versus host disease only (Level A I). Aerosolized liposomal amphotericin B is recommended during prolonged neutropenia (Level B II). There is moderate evidence of voriconazole for prophylaxis of invasive fungal infections measured and compared eighty days after end of prophylaxis [25].

Physicians are now headed with the management of suspected fungal infections in febrile neutropenic patients undergoing posaconazole prophylaxis. Posaconazole prophylaxis failure was rare in the trials that led to the approval of posaconazole. Fever refractory to antibiotics despite posaconazole prophylaxis remains a common clinical scenario during the course of leukemia treatment however. Wait and see or change of antifungal treatment is the question [26]. IFI under posaconazole prophylaxis were diagnosed in $2 \%$ and $5 \%$ of patients with AML and allogeneic HSCT-recipients with GvHD, respectively. A posaconazole trough threshold of $0.5 \mu \mathrm{g} / \mathrm{ml}$ has been proposed for optimized antifungal prevention. Statistically different posaconazole plasma concentrations have been observed under various conditions. There is a matter of debate what is clinically relevant [27]. Thus, drug monitoring of triazole serum concentrations may become important to minimize toxicity and ensure efficacy [28, 29, 30]. Kohl et al elucidated factors influencing the pharmacokinetics of posaconazole administered in HSCT-recipients for prophylaxis. Among the covariates tested, significant effects were found for age (decrease in the volume of distribution of 123 liters per year of age) and the presence of diarrhea (59\% loss of 
bioavailability). A basis for prediction of the mean posaconazole concentrations in allogeneic SCT recipients with hematological malignancies is provided for a given dose. Corresponding adjustments of the starting dose according to the presence of diarrhea, and according to age appear to be justified [31].

A French trial retrospectively analyzed low posaconazole plasma concentrations (PPC) defined as a concentration lower than $500 \mathrm{ng} / \mathrm{ml}$. Fifty-four patients were included: 36 receiving prophylactic (200 $\mathrm{mg}$ three times a day) and 18 receiving curative (400 mg twice a day) doses of posaconazole. The prevalence of low PPCs was 44\% (16/36) in the prophylaxis group and $22 \%(4 / 18)$ in the curative-treatment group. In the prophylaxis group, low PPCs tended to be more frequent in cases of digestive disease $(62.5 \%$ versus $30 \% ; \mathrm{P}=0.051)$ and were significantly more frequent among patients with diarrhea $(71.4 \%$ versus $27 \% ; \mathrm{P}=0.009)$ or mucositis $(100 \%$ versus $33 \% ; \mathrm{P}=$ 0.004). In the curative-treatment group, low PPCs were significantly more frequent in cases of diarrhea (75\% versus $7 \% ; \mathrm{P}=0.018$ ) [32]. Taken together the results of these two trials suggest that therapeutic drug monitoring of posaconazole is mandatory in immunosuppressed patients, at least in those with gastrointestinal disorders and the administration of proton pump inhibitors. Patients with fever during posaconazole prophylaxis might be switched to an intravenous broad spectrum antimycotic, at least in cases of mucositis and diarrhea. Breakthrough fungal infections caused by Zygomycosis should be taken into account, in particular for those patients receiving voriconazole for prophylaxis [33]. Case reports with proven pneumonia due to Rhizopus after long standing posaconazole prophylaxis have also been published recently [34]. Fasting serum levels were in a range of $691-904 \mathrm{ng} / \mathrm{mL}$ in one of the cases [35]. The presented cases had severe graft-versus-host disease after allogeneic stem cell transplantation associated with an impaired intestinal absorption of posaconazole.

\section{Management of Invasive AspergiLlosis}

Patients at highest risk are those with hematological malignancies and severe neutropenia. Emerging data have shown that solid organ transplant recipients, chronic obstructive pulmonary disease and patients in the intensive care unit receiving steroids might have a risk for invasive aspergillosis too. Non-neutropenic, non-hematological patients now account for up to $40 \%$ of all those with invasive aspergillosis. For these latter patients there is less knowledge of the management. The approaches that have been proven to be effective in the neutropenic host may also benefit in these patient groups. Early and effective treatment with maximum tolerable dosages of antifungal agents is the backbone of treatment.

For decades Amphotericin B desoxycholate has been the only option for invasive aspergillosis until the early 1990s. The introduction of voriconazole has been a major progress. Voriconazole has a broad antifungal efficacy, including Aspergillus species. Patients with definitive or probable invasive aspergillosis were studied in an open randomized trial that compared voriconazole $(2 \mathrm{x} 6 \mathrm{mg} / \mathrm{kg}$ on day 1 and $2 \times 4 \mathrm{mg} / \mathrm{kg} / \mathrm{d}$ for seven days i.v. followed by p.o treatment) with amphotericin B desoxycholate $(1-1,5 \mathrm{mg} / \mathrm{kg} / \mathrm{d})$. At week 12 response was observed in $52.8 \%$ in the voriconazole and $31.6 \%$ in the amphotericin B group. Patients in the voriconazole arm had a significant improved survival at week 12 (70.8\% vs $57.9 \%)$. Toxicity was lower for patients receiving voriconazole beside visual disturbances [36]. Since that trial voriconazole has been adopted gold standard for the treatment of invasive Aspergillosis. One might argue that amphotericin $\mathrm{B}$ desoxycholat may not have been the optimal competitor, since amphotericin B could be administered a median of 10 days only [37]. Amphotericin B desoxycholate was the only approved drug for first-line treatment at that time the trial was designed, however. Treatment with voriconazole proved to be effective even in patients with disseminated aspergillosis infections. Moreover, voriconazole penetrates the central nervous system (CNS). Patients with invasive aspergillosis of the CNS are known to have a very bad prognosis with a mortality of $90 \%$. Voriconazole showed superior efficacy and survival with a response in $35 \%$ of patients (survival 31\%) [38]. Although voriconazole is well absorbed from the gut there has been reported a large inter-individual variability in voriconazole through blood levels. Voriconazole blood levels of $>1 \mathrm{mg} / \mathrm{L}$ are considered a minimum inhibitory concentration. Lack of response has been observed in patients with voriconazole levels $<1 \mathrm{mg} / \mathrm{L}$ $[39,40]$. Efficacy against invasive fungal infections was documented after increasing the voriconazole dosage. Therefore, therapeutic drug monitoring of voriconazole is recommended in particular for those patients not responding under oral voriconazole treatment. Higher voriconazole levels $(>5.5 \mathrm{mg} / \mathrm{L}$ ) in contrast are associated with increased adverse events, in particular encephalopathy due to voriconazole. Co-medication with omeprazole has been observed with voriconazole accumulation. In these cases discontinuation of therapy resulted in complete neurological recovery.

\section{Liposomal AmpHotericin B}

Treatment of invasive aspergillosis needs maximum tolerable dosages of antifungal drugs. Liposomal amphotericin B achieves maximum plasma levels at a dosage of $10 \mathrm{mg} / \mathrm{kg}$ per day. Induction treatment of proven or probable invasive aspergillosis with high dose $(10 \mathrm{mg} / \mathrm{kg}) \mathrm{LAmB}$ was compared in a doubleblind trial. 201 Patients with mold infection were randomized to receive LAmB at either 3 or $10 \mathrm{mg} / \mathrm{kg}$ per day for 14 days, followed by $3 \mathrm{mg} / \mathrm{kg}$ per day. The primary end point was complete or partial response. A favorable response was achieved in $50 \%$ and $46 \%$ of patients in the 3 - and $10-\mathrm{mg} / \mathrm{kg}$ groups, respectively $(\mathrm{P}>.05)$. Survival rates at 12 weeks were $72 \%$ and $59 \%(\mathrm{P}>.05)$. Significantly higher rates of nephrotoxicity and hypokalemia were seen in the high-dose group. Liposomal Amphotericin B has been approved for first-line treatment of invasive fungal infection [41]. The AmBiLoad trial was not a comparison to 
Table 3. First-line Treatment of Invasive Aspergillosis: Prospective Controlled Trials.

\begin{tabular}{|c|c|c|c|c|c|c|}
\hline Study & $\mathrm{N}=$ & Design & Treatment & $\begin{array}{l}\text { MDST } \\
\text { (range) }\end{array}$ & $\begin{array}{l}\text { Response } \\
(\mathrm{CR}+\mathrm{PR})\end{array}$ & $\begin{array}{l}\text { Survival } \\
\text { week } 12\end{array}$ \\
\hline Herbrecht & \multirow[t]{2}{*}{277} & \multirow[t]{2}{*}{ op, rd } & AmB Desoxycholate $1-1.5 \mathrm{mg} / \mathrm{kg}$ & $10(1-84)$ & $31.6 \%$ & $57.9 \%$ \\
\hline 2002 NEJM & & & Vori $2 \times 6 \mathrm{mg} / \mathrm{kg} \mathrm{d} 1$ and $2 \times 4 \mathrm{mg} / \mathrm{kg} \mathrm{d} 2+$ i.v.* & $77(2-84)$ & $52.8 \%$ & $70.8 \%$ \\
\hline Cornely & \multirow[t]{2}{*}{201} & \multirow[t]{2}{*}{$\mathrm{db}, \mathrm{rd}$} & $\mathrm{LAmB} 3 \mathrm{mg} / \mathrm{kg}(\mathrm{d} 1-14) \quad \mathbf{Z}$ & $14(1-60)$ & $50 \%$ & $72 \%$ \\
\hline 2007 CID & & & $\mathrm{LAmB} 10 \mathrm{mg} / \mathrm{kg}(\mathrm{d} 1-14) \boldsymbol{\zeta}^{\text {of }}$ & $15(1-57)$ & $46 \%$ & $59 \%$ \\
\hline Herbrecht 2010 BMT ** & 24 & op, sa & Caspofungin $70 \mathrm{mg} \mathrm{d} 1 / 50 \mathrm{mg} \mathrm{d} 2+$ & 24 & $33 \%$ & $50 \%$ \\
\hline Viscoli 2009JAC \# & 61 & op, sa & Caspofungin $70 \mathrm{mg} \mathrm{d} 1 / 50 \mathrm{mg} \mathrm{d} 2+$ & $15(3-84)$ & $33 \%$ & $53 \%$ \\
\hline
\end{tabular}

Abbreviations: op $=$ open, $\mathrm{rd}=$ randomized, $\mathrm{db}=$ double blind, $\mathrm{sa}=$ single arm, $\mathrm{MDST}=$ Median duration of study drug treatment in days, * a switch to oral voriconazole was allowed after day 7 , **allogeneic cohort of patients, \# hematological malignancies and autologous transplantation

voriconazole what has to be taken into account. Although response and survival rates were in the range of voriconazole for first-line therapy of invasive aspergillosis. A head to head comparison of both drugs for efficacy against invasive aspergillosis is lacking.

\section{ECHINOCANDINS FOR THE MANAGEMENT OF INVASIVE ASPERGILLOSIS}

Echinocandins are approved for 2nd-line treatment of invasive Aspergillosis being refractory or intolerant against 1st-line drugs [42]. A small open label Phase II study ( $\mathrm{n}=61)$ evaluated caspofungin as first-line therapy for invasive aspergillosis in hematological diseases with $85 \%$ of patients being neutropenic at enrolment. At the end of treatment one patient had a complete and 19 patients a partial response [success rate 33\% (20/61)] with 31 patients (51\%) showing disease progression. The 12 -week survival rate was 53\% (32/60) [43]. Caspofungin has not been compared to voriconazole for first-line treatment of invasive aspergillosis (see Table 3). In addition, caspofungin is an active first-line therapy for Candida infections in both, neutropenic and non-neutropenic patients [44, 45].

Micafungin and anidulafungin are also approved for the treatment of invasive Candidiasis with fewer data in hematological patients $[46,47,48]$. However, both drugs have not been proven to be effective for firstline treatment for invasive aspergillosis whereas some data are available on micafungin for refractory aspergillosis [49].

\section{Posaconazole for Treatment of Invasive AsPERGILLOSIS}

Currently there are no data for the first-line treatment from randomized trials. The posaconazole salvage study was conducted in patients with refractory or intolerant invasive mycoses [50]. These patients were not randomized in a controlled clinical trial but were included as a prespecified control group in the original study plan using retrospective data. A data review committee of 15 experts in antifungal therapy and 2 radiologists assessed posaconazole-treated subjects and control subjects in a parallel, blinded manner using predefined methods to assess evaluability and out- come. Cases of aspergillosis deemed evaluable by a blinded data review committee included 107 posaconazole recipients and 86 control subjects (modified intent-to-treat population). The overall success rate was $42 \%$ for posaconazole recipients and $26 \%$ for control subjects $(\mathrm{P}=.006)$. The 2 nd-line data of posaconazole treatment of IA showed efficacy in patients failing first-line treatment. These data do not allow to estimate efficacy in the first-line treatment of IA with posaconazole.

\section{ITRACONAZOLE FOR INVASIVE ASPERGILLOSIS}

Intravenous itraconazole circumvents problems with intestinal absorption. Intravenous followed by oral itraconazole has been reported for the treatment of invasive aspergillosis in a non-randomized trial [51]. An open, international, multicenter trial evaluated the efficacy and safety of i.v. followed by oral itraconazole capsules in patients with invasive pulmonary aspergillosis. Patients received $200 \mathrm{mg}$ itraconazole by an iv infusion over 60-90 min every $12 \mathrm{~h}$ for the first 2 days. For the following 12 days, $200 \mathrm{mg}$ itraconazole was administered by iv infusion once daily. Oral itraconazole capsules (200-mg) were then administered twice daily from weeks 3-14. Median iv itraconazole was 14 days with mean trough concentrations of itraconazole after 2 and 14 days 670 and $850 \mathrm{ng} / \mathrm{mL}$, respectively. A complete or partial response was seen at the last on-treatment assessment in $15(48 \%)$ of 31 patients, with $6(19 \%)$ showing stable disease.

\section{Management of InVAsive AspergiLlosis WITH GM-MONITORING}

Since the widespread use of posaconazole for prophylaxis in high risk hematological malignancies management of suspected invasive aspergillosis has raised new questions in particular, the definition of breakthrough aspergillus infection. Is this refractory fever under posaconazole prophylaxis, or fever under posaconazole and new lung infiltrates?

Galactomannan (GM) screening has been introduced in recent years [52]. We have learned to use this diagnostic tool for early detection of invasive aspergillosis [53]. These data were obtained prior to the 
introduction of posaconazole for prophylaxis. The role of quantitative GM-serum levels in patients under prophylaxis with posaconazole remains a matter of debate. Different clinical scenarios may be encountered: patients who develop new fever without any new lung infiltrates, patients with newly diagnosed febrile episodes and new lung infiltrates in the absence of a positive GM-assay, and patients with fever plus/minus new lung infiltrates and a positive serum GM. Monitoring aspergillosis response to therapy by conventional clinical, radiological, and microbiological methods remains challenging, because it includes subjective and nonspecific signs, symptoms of infection, and interpretation of radiological findings that are neither standardized nor validated. A decrease of GM in patients with proven aspergillosis might be helpful for the interpretation of radiological findings during the course of the disease. A normalization of GM is not enough to terminate antifungal treatment.

If failure of posaconazole prophylaxis is suspected, a thorough clinical investigation is necessary. This includes control of compliance with oral posaconazole ingestion, timepoint of administration (Posaconazole administered at dosages of $600-800 \mathrm{mg} /$ day in divided doses with food) and signs and symptoms of diarrhea or gastrointestinal GvHD. Determining posaconazole serum/plasma concentration seems to be helpful but the assessment of serum levels is not a routine in daily practice. If fever is the only symptom in a patient with adequate posaconazole serum levels, a wait and see policy is justified.

By contrast, posaconazole prophylaxis may be terminated in patients with a worsening clinical condition. In particular breakthrough infections related to Zygomycosis have to be ruled out or should be taken into account. Awareness of the local epidemiology may be important [54]. For patients developing new lung infiltrates or symptoms of acute rhinosinusitis while being on posaconazole for IFI prophylaxis a switch to other licensed antifungals should be considered. There are no data from clinical trials supporting the use of voriconazole or an echinocandin in patients who develop fever under posaconazole prophylaxis. Of note, voriconazole and the echinocandins have no clinical efficacy against Zygomycosis. A combination therapy would be of particular interest, e.g. posaconazole plus liposomal amphotericin B or an echinocandin. Data from controlled clinical trials supporting this concept may be available by the end of 2011. At present a change to lipid-based amphotericin B seems to be reasonable for patients with posaconazole prophylaxis with a worsening clinical condition that is suspected to be related to an invasive aspergillosis. Amphotericin B has a broader spectrum of activity compared to azoles and echinocandins. Furthermore, amphotericin B covers most Candida species and has activity against Trichosporum, Blastomyces, Cryptococci as well as Zygomycosis.

\section{Combination of Antifungal Agents Against Invasive Aspergillosis}

Combined, simultaneous or sequential antifungal therapy are often been considered an appropriate option for salvage treatment of invasive aspergillosis. Preliminary data suggest that the combination of azoles or LAmB and echinocandins may increase activity against refractory IA $[55,56]$. Anidulafungin belongs to the echinocandin family. A randomized double-blind placebo controlled Phase III trial compares efficacy of voriconazole plus anidulafungin or placebo. This trial has stopped enrolment. Data are suspected in late 2011.

It should be emphasized that antifungal prophylaxis will remain a standard approach in patients at high risk for invasive fungal infections while meticulous clinical judgement and treatment of febrile neutropenic episodes remain necessary. New aszoles, lipid based amphotericin and the echinocandins have improved the management of invasive aspergillosis. Patients with invasive aspergillosis must closely be monitored. A switch of the antifungal regimen may help to achieve a better outcome of patients worsening under posaconazole prophylaxis. The question which antifungal should be used has to be answered by well-designed prospective clinical trials.

\section{REFERENCES}

1. Lehrnbecher T, Frank C, Engels K, et al. Trends in the postmortem epidemiolgy at a university hospital. J Infect. 2010 Sep;61(3):259-65

2. Stevens DA, Kan VL, et al. Practice guidelines for diseases caused by Aspergillus. Clin. Infect. Dis. 2000; 30:696709.

3. Rex JH, Walsh TJ, Anaissie EA. Fungal infections in iatrogenically compromised hosts. Adv. Intern. Med. 1998; 43:321-371

4. Rex JH, Walsh TJ, Sobel JD, et al. Practice guidelines for the treatment of candidiasis. Clin. Infect. Dis. 2000; 30:662-678.

5. De Pauw B, Walsh TJ, Donnelly JP, et al. Revised definitions of invasive fungal disease from the European Organization for Research and Treatment of Cancer/Invasive Fungal Infections Cooperative Group and the National Institute of Allergy and Infectious Diseases Mycoses Study Group (EORTC/MSG) Consensus Group. Clin Infect Dis. 2008 Jun 15;46(12):1813-21.

6. Maertens J, Van Eldere J, Verhaegen J, et al. Use of circulating galactomannan screening for early diagnosis of invasive aspergillosis in allogeneic stem cell transplant recipients. J Infec Dis. 2002 186:1297-1306

7. Cornely OA, Ullmann AJ, Karthaus M. Evidence-based assessment of primary antifungal prophylaxis in patients with hematologic malignancies. Blood. 2003 May 1;101(9):336572. Epub 2002 Aug 29.

8. Eriksson U, Seifert B, Schaffner A. Comparison of effects of amphotericin B deoxycholate infused over 4 or 24 hours: randomised controlled trial. BMJ. 2001 Mar 10;322(7286):579-82.

9. Infectious Diseases Working Party (AGIHO) of the German Society of Hematology and Oncology (DGHO), Böhme A, Ruhnke M, Buchheidt D,et al. Treatment of invasive fungal infections in cancer patients--recommendations of the Infectious Diseases Working Party (AGIHO) of the German Society of Hematology and Oncology (DGHO). Ann Hematol. 2009 Feb;88(2):97-110.

10. Walsh TJ, Anaissie EJ, Denning DW, et al. Treatment of aspergillosis: Clinical practice guidelines of the IDSA. CID 2008; 46: 327-60.

11. Walsh TJ, Finberg RW, Arndt C, et al. Liposomal amphotericin $\mathrm{B}$ for empirical therapy in patients with persistent fever and neutropenia. National Institute of Allergy and Infectious Diseases Mycoses Study Group. N Engl J Med. 1999 Mar 11;340(10):764-71. 
12. Prentice HG, Hann IM, Herbrecht R, et al. A randomized comparison of liposomal versus conventional amphotericin B for the treatment of pyrexia of unknown origin in neutropenic patients. Br J Haematol. 1997 Sep;98(3): 711-8.

13. Walsh TJ, Teppler H, Donowitz GR,et al. Caspofungin versus liposomal amphotericin B for empirical antifungal therapy in patients with persistent fever and neutropenia. N Engl J Med. 2004 Sep 30;351(14):1391-402.

14. Goodman JL, Winston DJ, Greenfield RA, et al. A controlled trial of fluconazole to prevent fungal infections in patients undergoing bone marrow transplantation. $\mathrm{N}$ Engl J Med. 1992;326:845-851

15. Slavin MA, Osborne B, Adams R, et al. Efficacy and safety of fluconazole prophylaxis for fungal infections after marrow transplantation---a prospective, randomized, doubleblind study. J Infect Dis. 1995;171:1545-1552

16. Marr KA, Seidel K, Slavin MA,et al. Prolonged fluconazole prophylaxis is associated with persistent protection against candidiasis-related death in allogeneic marrow transplant recipients: long-term follow-up of a randomized, placebocontrolled trial. Blood. 2000 Sep 15;96(6):2055-61.

17. Cornely OA, Maertens J, Winston DJ, et al. Posaconazole vs. fluconazole or itraconazole prophylaxis in patients with neutropenia. N Engl J Med. 2007 Jan 25;356(4):348-59.

18. Ullmann AJ, Lipton JH, Vesole DH, et al. Posaconazole or fluconazole for prophylaxis in severe graft-versus-host disease. N Engl J Med. 2007 Jan 25;356(4):335-47. Erratum in: N Engl J Med. 2007 Jul 26;357(4):428.

19. van Burik JA; Ratanatharathorn V, Stepan DE, Miller CB, Lipton JH, Vesole DH, Bunin N, Wall DA; Hiemenz JW; Satoi Y, Lee JM, Walsh TJ; National Institute of Allergy and Infectious Diseases Mycoses Study Group. Micafungin versus fluconazole for prophylaxis against invasive fungal infections during neutropenia in patients undergoing hematopoietic stem cell transplantation. Clin Infect Dis. 2004 Nov 15;39(10):1407-16

20. Penack O, Schwartz S, Martus P, Reinwald M, SchmidtHieber M, Thiel E, Blau IW. Low-dose liposomal amphotericin $\mathrm{B}$ in the prevention of invasive fungal infections in patients with prolonged neutropenia: results from a randomized, single-center trial. Ann Oncol 2006 Aug;17(8): 1306-12

21. Rijnders BJ, Cornelissen JJ, Slobbe L, Becker MJ, Doorduijn JK, Hop WC, Ruijgrok EJ, Löwenberg B, Vulto A, Lugtenburg PJ, de Marie S. Aerosolized liposomal amphotericin B for the prevention of invasive pulmonary aspergillosis during prolonged neutropenia: a randomized, placebo-controlled trial. Clin Infect Dis 2008; 46(9): 1401-8

22. Vehreschild JJ, Böhme A, Buchheidt D, et al. A doubleblind trial on prophylactic voriconazole (VRC) or placebo during induction chemotherapy for acute myelogenous leukaemia (AML). J Infect. 2007 Nov;55(5):445-9.

23. Wingard JR, Carter SL, Walsh TJ, et.al. Blood an Marrow Transplant Clinical Trials Network. Randomized, doubleblind trial of fluconazole versus voriconazole for prevention of invasive fungal infection after allogeneic hematopoietic cell transplantation. Blood 2010; $116(24): 5111-8$

24. Prentice AG, Glasmacher A, Djulbegovic B. In metaanalysis itraconazole is superior to fluconazole for prophylaxis of systemic fungal infection in the treamtent of hematological malignancy. Br J Haematol 2005; 131(1):22-8

25. Cornely OA, Böhme A, Buchheidt D, et al.. Primary prophylaxis of invasive fungal infections in patients with hematologic malignancies. Recommendations of the Infectious Diseases Working Party of the German Society for Haematology and Oncology. Haematologica 2009; 94(1):113-22

26. Karthaus M, Hentrich M. Wait and see or rush and switch? New questions for the management of patients with febrile neutropenia receiving antifungal prophylaxis. Mycoses. 2011 Jan; 54 (Suppl 1): 1-6
27. Krishna G, AbuTarif M, Xuan F, et al. Pharmakokinetics of oral posaconazole in neutropenic patients receiving chemotherapy for acute myelogenous leukemia or myleodysplastic syndrome. Pharmacotherapy. 2008; 28(10): 122332.

28. Ullmann AJ, Cornely OA, Burchardt A, et al. Pharmacokinetics, safety, and efficacy of posaconazole in patients with persistent febrile neutropenia or refractory invasive fungal infection. Antimicrob Agents Chemother. 2006 Feb;50(2): 658-66.

29. Lipp HP. Posaconazole:clinical pharmacokinetics and drug interactions. Mycoses. 2011 Jan(Suppl 1): 32-8.

30. Shields RK, Clancy CJ, Vadnerkar A, et al. Posaconazole serum concentrations among cardiothoracic transplant reciepients: Factors influencing levels and correlation with clinical response. Antimicrob Agents Chemother. 2010 Dec 2010 [Epub ahead of print]

31. Kohl V, Müller C, Cornely OA, et al. Factors influencing pharmacokinetics of prophylactic posaconazole in patients undergoing allogeneic stem cell transplantation. Antimicrob Agents Chemother. 2010 Jan;54(1):207-12.

32. Lebeaux D, Lanternier F, Elie C, et al. Therapeutic drug monitoring of posaconazole: a monocentric study with 54 adults. Antimicrob Agents Chemother. 2009 Dec;53(12): 5224-9. Epub 2009 Sep 14.

33. Trifilio SM, Bennett CL, Yarnold PR, et al. Breakthrough zygomycosis after voriconazole administration among patients with hematologic malignancies who receive hematopoietic stem-cell transplants or intensive chemotherapy. Bone Marrow Transplant. 2007 Apr;39(7):425-9.

34. Lekakis LJ, Lawson A, Prante J, et al. Fatal rhizopus pneumonia in allogeneic stem cell transplant patients despite posaconazole prophylaxis: two cases and review of the literature. Biol Blood Marrow Transplant. 2009 Aug;15(8): 991-5. .

35. Mousset S, Bug G, Heinz WJ, et al. Breakthrough zygomycosis on posaconazole prophylaxis after allogeneic stem cell transplantation. Transpl Infect Dis. 2010 Jun;12(3): 261-4

36. Herbrecht R, Denning DW, Patterson TF, et al. Voriconazole versus amphotericin $\mathrm{B}$ for primary therapy of invasive aspergillosis. N Engl J Med. 2002 Aug 8;347(6):408-15.

37. Karthaus M. Voriconazole versus amphotericin B for invasive aspergillosis. N Engl J Med. 2002 Dec 19;347(25): 2080-1.

38. Schwartz S, Ruhnke M, Ribaud P, et al. Improved outcome in central nervous system aspergillosis using voriconazole treatment. Blood 2005; 106(8):2641-5.

39. Pascual A, Calandra T, Bolay S, et 1 . Voriconazole therapeutic drug monitoring in patients with invasive mycoses improves efficacy and safety outcome. Clin Infec Dis 2008; 46(2):201-11

40. Trifilio S, Pennick G, Pi J, Zook J, et al. Monitoring voriconzole levels may be necessary to avoid subtherapeutic levels in hematopoietic stem cell recipients. Cancer 2007; 109(8):1532-5.

41. Cornely OA, Maertens J, Bresnik M, et al. Liposomal amphotericin B as initial therapy for invasive mold infection: a randomized trial comparing a high-loading dose regimen with standard dosing (AmBiLoad trial). Clin Infect Dis. 2007 May 15;44(10):1289-97.

42. Maertens J, Raad I, Petrikkos G, et al. Efficacy and safety of caspofungin for treatment of invasive aspergillosis in patients refractory to or intolerant of conventional antifungal therapy. Clin Infect Dis. 2004 Dec 1;39(11):1563-71. Epub 2004 Nov 9.

43. Viscoli C, Herbrecht R, Akan H, et al. An EORTC Phase II study of caspofungin as first-line therapy of invasive aspergillosis in haematological patients. J Antimicrob Chemother. 2009 Dec;64(6):1274-81. Epub 2009 Oct 19.

44. Mora-Duarte J, Betts R, Rotstein C, et al.Comparison of caspofungin and amphotericin B for invasive candidiasis. N Engl J Med. 2002 Dec 19;347(25):2020-9. 
45. Betts R, Glasmacher A, Maertens J, et al. Efficacy of caspofungin against invasive Candida or invasive Aspergillus infections in neutropenic patients. Cancer. 2006 Jan 15;106(2):466-73.

46. Kuse ER, Chetchotisakd P, da Cunha CA, et al. Micafungin versus liposomal amphotericin B for candidaemia and invasive candidosis: a phase III randomised double-blind trial.Lancet. 2007 May 5;369(9572):1519-27.

47. Pappas PG, Rotstein CM, Betts RF, et al. Micafungin versus caspofungin for treatment of candidemia and other forms of invasive candidiasis. Clin Infect Dis. 2007 Oct 1;45(7):883-93.

48. Reboli AC, Rotstein C, Pappas PG, et al. Anidulafungin versus fluconazole for invasive candidiasis. $\mathrm{N}$ Engl J Med. 2007 Jun 14;356(24):2472-82.

49. Denning DW, Marr KA, Lau WM, et al. Micafungin (FK463), alone or in combination with other systemic antifungal agents, for the treatment of acute invasive aspergillosis. J Infect. 2006 Nov;53(5):337-49.

50. Walsh TJ, Raad I, Patterson TF, et al. Treatment of invasive aspergillosis with posaconazole in patients who are refractory to or intolerant of conventional therapy: an externally controlled trial. Clin Infect Dis. 2007 Jan 1;44(1):2-12.

51. Caillot D, Bassaris H, McGeer A, et al. Intravenous itraconazole followed by oral itraconazole in the treatment of pulmonary aspergillosis in patients with hematological malignancies, chronic granulomatous disease or AIDS . Clin Infect Dis. 2010; 33(8):e83-90.

52. Marr KA, Balajee SA, McLaughlin L, et al. Detection of galactomannan antigenemia by enzyme immunoassay for the diagnosis of invasive aspergillosis: variables that affect performance. J Infect Dis. 2004 Aug 1;190(3):641-9.
53. Maertens JA, Klont R, Masson C, et al. Optimization of the cutoff value for the Aspergillus double-sandwich enzyme immunoassay. Clin Infect Dis. 2007 May 15;44(10): 1329-36.

54. Rickerts V, Böhme A, Viertel A, et al. Cluster of pulmonary infections caused by Cunninghamella bertholletiae in immunocompromised patients. Clin Infect Dis. 2000 Oct; 31(4):910-3.

55. Kontoyiannis DP, Ratanatharathorn V, Young JA, et al. Micafungin alone or in combination with other systemic antifungal therapies in hematopoietic stem cell transplant recipients with invasive aspergillosis. Transpl Infect Dis. 2009 Feb;11(1):89-93.

56. Thomas A, Korb V, Guillemain R, et al. Clinical outcomes of lung-transplant recipients treated by voriconazole and caspofungin combination in aspergillosis. J Clin Pharm Ther. 2010 Feb;35(1):49-53.

Received: February 6, 2011 / Accepted: February 22, 2011

Address for correspondence:

Prof. Dr. Meinolf Karthaus

Klinikum Neuperlach

Oskar-Maria-Graf Ring 51

D-81737 München

Germany

Phone: +8967942651

Fax: $\quad+8967942448$

E-mail: meinolf.karthaus@klinikum-muenchen.de 